\title{
Enhancing the High Voltage XLPE Cable Insulation Characteristics Using Functionalized $\mathrm{TiO}_{2}$ Nanoparticles
}

\author{
Abdelrahman Said ${ }^{1}$, Amira Gamal Nawar ${ }^{1,2, ~ *, ~ E l s a y e d ~ A l a a ~ E l d e s o k y ~}{ }^{3}$, Samir Kamel ${ }^{4}$, \\ Mousa Awdallah Abd-Allah ${ }^{1}$ \\ ${ }^{1}$ Electrical Engineering Department, Shoubra Faculty of Engineering, Benha University, Cairo, Egypt \\ ${ }^{2}$ Higher Institute for Engineering and Modern Technology, Marg, Cairo, Egypt \\ ${ }^{3}$ Polymer and Pigment Department, National Research Centre, Giza, Egypt \\ ${ }^{4}$ Cellulose and Paper Department, National Research Centre, Giza, Egypt
}

\section{Email address:}

abdelrahman.ghoniem@feng.bu.edu.eg (A. Said), eng_amira.2009@yahoo.com (A. G. Nawar), alaa_chemist@yahoo.com (E. A. Eldesoky), samirki@yahoo.com (S. Kamel), mousa_abdulah@yahoo.co (M. A. Abd-Allah)

${ }^{*}$ Corresponding author

\section{To cite this article:}

Abdelrahman Said, Amira Gamal. Nawar, Elsayed Alaa. Eldesoky, Samir Kamel, Mousa Awdallah. Abd-Allah. Enhancing the High Voltage XLPE Cable Insulation Characteristics Using Functionalized $\mathrm{TiO}_{2}$ Nanoparticles. American Journal of Polymer Science and Technology. Vol. 6, No. 3, 2020, pp. 21-31. doi: 10.11648/j.ajpst.20200603.11

Received: August 30, 2020; Accepted: September 14, 2020; Published: October 13, 2020

\begin{abstract}
The current research aims to study the influence of loading Titanium Dioxide $\left(\mathrm{TiO}_{2}\right)$ nanoparticles on the dielectric, thermal and mechanical properties of the commercial Cross-Linked Polyethylene (XLPE) used as the main insulation in power cables. Using the concept of composite, $\mathrm{XLPE} / \mathrm{TiO}_{2}$ nanocomposites samples were prepared by the melt blending method with different ratios of nanoparticles $(0.5,2,3.5$ and $5 \% \mathrm{wt} / \mathrm{wt})$. The surface treatment of $\mathrm{TiO}_{2}$ nanoparticles was carried out to reduce the agglomeration of $\mathrm{TiO}_{2}$ nanoparticles inside the XLPE. The morphology of the prepared samples was studied by X-ray Diffraction (XRD) and the dispersion of nanoparticles in the XLPE polymer matrix is checked using Field Emission Scanning Electron Microscopy (FE-SEM). Thermal analysis test for all samples have been investigated. The dielectric properties, such as dielectric constant $\left(\varepsilon_{\mathrm{r}}\right)$ and loss tangent $(\tan \delta)$ for $\mathrm{XLPE} / \mathrm{TiO}_{2}$ nanocomposites were measured under frequencies ranging from $1 \mathrm{~Hz}$ to $1 \mathrm{MHz}$. AC Breakdown Voltage (AC-BDV) was also measured using a controlled high voltage testing transformer $(50 \mathrm{~Hz})$ under sphere-to-sphere field. The mechanical properties were evaluated by performing the tensile test and tensile strength and elongation values were measured. It was found that nanocomposites with functionalized $\mathrm{TiO}_{2}$ exhibited better dielectric, thermal and mechanical properties compared to nanocomposites with nonfunctionalized $\mathrm{TiO}_{2}$.
\end{abstract}

Keywords: XLPE, Nanocomposites, Titanium Nanoparticles, Electrical, Thermal, and Mechanical Properties

\section{Introduction}

The excellent characteristics of polymers, such as reliability, availability, low cost, light weight, easy fabrications, and appropriate processing ability, support their using as the main insulation in high voltage cables [1]. Polyethylene (PE) is the most important polyolefin type because of its superior properties; since it is a semi-crystalline polymer with a low cost and easy to manufacture and fabricate. Moreover, it has a wide array of engineering properties such as toughness, low coefficient of friction, chemical corrosion, and high resistance, and near-zero moisture absorption [2]. All these unique characteristics resulted in an ideal material for many applications especially in electrical insulation. Crosslinked Polyethylene (XLPE) is one shape from PE shapes. These polymers are widely used as electrical insulating materials; however, their high properties can be reduced over time due to different aging processes such as exposure to heat, humidity and mechanical stress [3-5]. So, there is still a need to further develop the insulation properties of these materials. One method for enhancing polymeric insulators behavior is using the concept of composite, inorganic particles were incorporated inside polymer matrix to produce what is called polymer composites $[6,7]$. In the previous decade, the design 
of composite materials involving either micro-scaled or nanoscaled inorganic and inorganic particles has gained great attention at power engineering to improve the properties of insulators dielectric, mechanical, and thermal [8-19]. The polymer composites were identified by their low working temperature, sensitivity to moisture and radiation also they have a high thermal expansion coefficient [20]. In many researches is seen that the particle type, size, weight, and surface of the polymer nanocomposites have an important role in its advantageous properties. Also, it is found that nanoparticles size introduced advantages over micron size because they increase the resistance to degradation and improve the thermo-mechanical properties without an unnoticeable decrease in dielectric strength $[21,22] . \mathrm{TiO}_{2}$ is inexpensive semiconductor have an excellent properties allow incorporation into polymeric matrices as adequate nanofiller to create new nanocomposites which have advanced behavior. $\mathrm{TiO}_{2}$ nanoparticles were chosen in this research because of their semi-conductive nature which acts well as charge traps and they are readily available [23]. This study aims to discuss the effect of incorporating nonfunctionalized and functionalized $\mathrm{TiO}_{2}$ with different ratios $(0.5,2,3.5$ and $5 \%$ $\mathrm{wt} / \mathrm{wt}$ ) inside the XLPE matrix by an industrial method and show it is an effect on the statistical characteristics of XLPE. To get optimum results, it is found that, the dispersion of nanoparticles within polymeric material should be homogenously distributed, and this can be done by treat and modify the nanoparticles surfaces chemically using silane coupling agents. This treatment of nanoparticles is carried out to make a conversion of their hydrophilic (inorganic) character into a hydrophobic (organic) character which leads to eliminating nanoparticles agglomeration and enhancing their distribution inside polymers matrices [24-27]. Transmission Electron Microscopy (TEM) is used to define the size of $\mathrm{TiO}_{2}$ nanoparticles. After preparation of $\mathrm{XLPE}^{\mathrm{T}} \mathrm{TiO}_{2}$ nanocomposites samples, the characterization can be studied with Field Emission Scanning Electron Microscopy (FE-SEM), thermal analysis and X-Ray Diffraction (XRD) techniques. Moreover, the dielectric properties such as dielectric strength, relative permittivity and loss tangent of the prepared samples were measured.

\section{Experimental Setup}

\subsection{Material}

CLNA-8141EHV is a crosslinked low-density polyethylene compound designed for extra high voltage power cable insulation that has a high degree of cleanliness was provided by El- Sewedy Electric Co., Egypt which has an extremely low level of contamination. Titanium Dioxide $\left(\mathrm{TiO}_{2}\right)$ nanoparticles were provided from sigma-Aldrich as titanium (IV) oxide CAS 13463-67-7, Mwt. 79.87, nanopowder, $99.5 \%$ purity. Methane sulfonic acid for surface activation of nanoparticles with $98 \%$ purity was purchased from LOBA Chemie. The coupling agent used is Gammaamino propyltriethoxy silane abbreviated as "amino silane" with purity $99 \%$ which was purchased from Momentive Inc. This study aims to discuss the effect of incorporating nonfunctionalized and functionalized $\mathrm{TiO}_{2}$ inside the XLPE matrix using an industrial method. The size of the nanoparticles will be measured. The mechanical characteristics and the dielectric properties such as relative permittivity, loss tangent and dielectric strength will be measured.

\subsection{Functionalization of Nanoparticle Materials}

The surface modification is performed through two sequential steps. The first step is called "surface activation" of the nanoparticles with hydroxyl groups through the acid etching method. The second step is called "surface modification" for the activated nanoparticles using amino silane as a coupling agent. In the first step, $10 \mathrm{~g}$ of $\mathrm{TiO}_{2}$ were stirred with $100 \mathrm{ml}$ of $10 \%$ methane-sulfonic acid at $110^{\circ} \mathrm{C}$ for 4 hours. After that, the powder was collected using Hitachi centrifuge at $1500 \mathrm{rpm}$ for $5 \mathrm{~min}$ and washed several times with de-ionized water then drying it for 24 hours at $120^{\circ} \mathrm{C}$ in a vacuum oven.

The second step is the functionalization of nanoparticles in which $3 \mathrm{~g}$ of the activated nanoparticles was added to $60 \mathrm{ml}$ of toluene in a round flask for sonication in a water bath sonicator (Elmasonic, S-60H) for $30 \mathrm{~min}$. at $70^{\circ} \mathrm{C}$ followed by stirring for $2 \mathrm{~h}$ at $500 \mathrm{rpm}$ and $70^{\circ} \mathrm{C}$. A solution of $10 \%$ (wt/wt) of amino silane in toluene was added dropwise and the stirring process was continued for 8 hours. Finally, the functionalized nanoparticles were collected and washed with isopropanol and dried in a vacuum oven at $120^{\circ} \mathrm{C}$ for 24 hours for further characterization [28-29].

\subsection{Preparation of XLPE Nanocomposites}

In our study, the $\mathrm{XLPE} / \mathrm{TiO}_{2}$ nanocomposites were prepared by the melt blending method which is the master batch method in the El-Sewedy Egy-tech laboratory. Each nanocomposite formulation consists of $150 \mathrm{~g}$ of XLPE pellets with different weights of either functionalized or nonfunctionalized $\mathrm{TiO}_{2}$ nanoparticles $(0.5,2,3.5$ and 5\% wt/ wt). XLPE/ $/ \mathrm{TiO}_{2}$ sheets, with dimension $20 \times 20 \mathrm{~cm}$ and $1.07-$ $2.2 \mathrm{~mm}$ thicknesses were prepared by as following; XLPE pellets and nanoparticles were mixed at $150^{\circ} \mathrm{C}$ with $80 \mathrm{rpm}$ for $15 \mathrm{~min}$ then annealed at $60^{\circ} \mathrm{C}$ for 24 hours. The mixture was blended in the two-roll mixer (Battaggion, MCC $150 * 300 / \mathrm{R}-\mathrm{o}$ ) at $150^{\circ} \mathrm{C}$ for $15 \mathrm{~min}$, with $30 \mathrm{rpm}$. The sample was pressed at $150^{\circ} \mathrm{C}$ under 200 bars for 15 min using GDBLaboratory hot/cold press (Gibitre instruments Co.).

\section{Characterization of Nanocomposites}

\subsection{Morphology Studies}

The morphology and size of $\mathrm{TiO}_{2}$ nanoparticles were studied using high-resolution Transmission Electron Microscope (TEM), the model type is (JEOL-JEM-2100). The images were recorded at a voltage of $120 \mathrm{kV}$. 


\subsection{Field Emission Scanning Electron Microscopy (FE-SEM)}

Field Emission Scanning Electron Microscopy (FE-SEM) (Quanta FEG 250, FEI-Inc.) was utilized for surface morphology investigation for some selected samples. The microscope was attached to the energy dispersive X-ray analysis (EDX) unit. The images have been recorded at a voltage of $10-15 \mathrm{kV}$.

\subsection{X-ray Diffraction (XRD)}

The XRD patterns of $\mathrm{XLPE} / \mathrm{TiO}_{2}$ nanocomposites were investigated on a Diano X-ray diffractometer using $\mathrm{CoK} \alpha$ radiation source energized at $45 \mathrm{kV}$ and a Philips X-ray diffractometer (PW 1930generator, PW 1820 goniometer) with $\mathrm{CuK}$ radiation source $(\lambda=0.15418 \mathrm{~nm})$, at a various angle range of $2 \theta$ between 10 to $80^{\circ}$ in reflection mode.

\subsection{Thermogravimetric Analysis (TGA)}

The thermal stability for $\mathrm{XLPE} / \mathrm{TiO}_{2}$ nanocomposites has been tested by thermogravimetric analysis (TGA, Shimadzu DTG-60, Japan) STA 6000 Perkin Elmer Analyzer. The analysis were done between $25^{\circ} \mathrm{C}$ to $900^{\circ} \mathrm{C}$ and the heating rate was $10^{\circ} \mathrm{C} / \mathrm{min}$ under argon.

\subsection{Dielectric Properties}

Relative permittivity and the loss factor were measured using Hioki 3532-50 LCR Hi Tester, frequency range of $1 \mathrm{~Hz}-1 \mathrm{MHz}$. The measurement of the breakdown strength was carried out using a sphere-to-sphere test cell energized from a test transformer $(400 \mathrm{~V} / 250 \mathrm{kV})$ according to ASTM (D149-09) standard. The measuring setup is modeled using a finite element method and the distribution of voltages and electric fields were determined in and around the different insulating samples.

\subsection{Mechanical Properties}

The mechanical properties such as elongation, and tensile

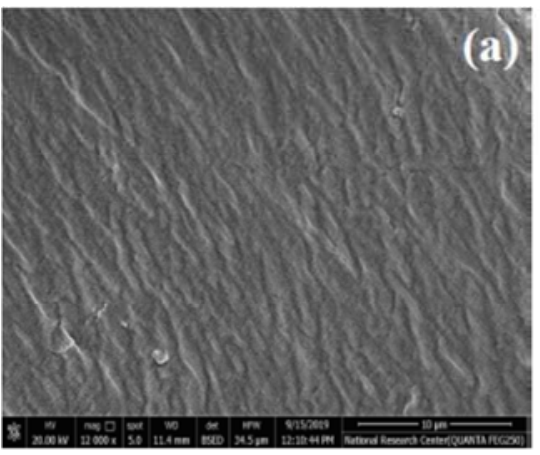

Figure 2. FE-SEM micrographs of (a) Cross-section area and (b) surface of the XLPE without nanoparticles.

The effect of amino silane surface treatments of $\mathrm{TiO}_{2}$ nanoparticles on the dispersion of $\mathrm{TiO}_{2}$ within the XLPE matrix can be achieved by comparing the FE-SEM micrographs for the cross-section area and the surface of the two nanocomposites one with nonfunctionalized and other with functionalized $\mathrm{TiO}_{2}$ as shown in Figure 3.

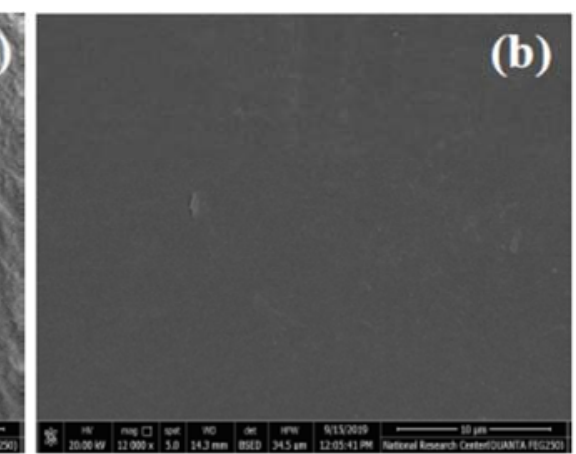

strength were measured by applying the tensile test using Plastic Micro-tensile Testing (ASTMD 1708-93 standards for micro tensile specimens). All mechanical properties are investigated at room temperature

\section{Results and Discussions}

\subsection{TEM Specification of Nanoparticles}

By using TEM, we can measure the size of the nanoparticles accurately. $\mathrm{TiO}_{2}$ nanoparticles average size was about (60-280) $\mathrm{nm}$ as shown in Figure 1.

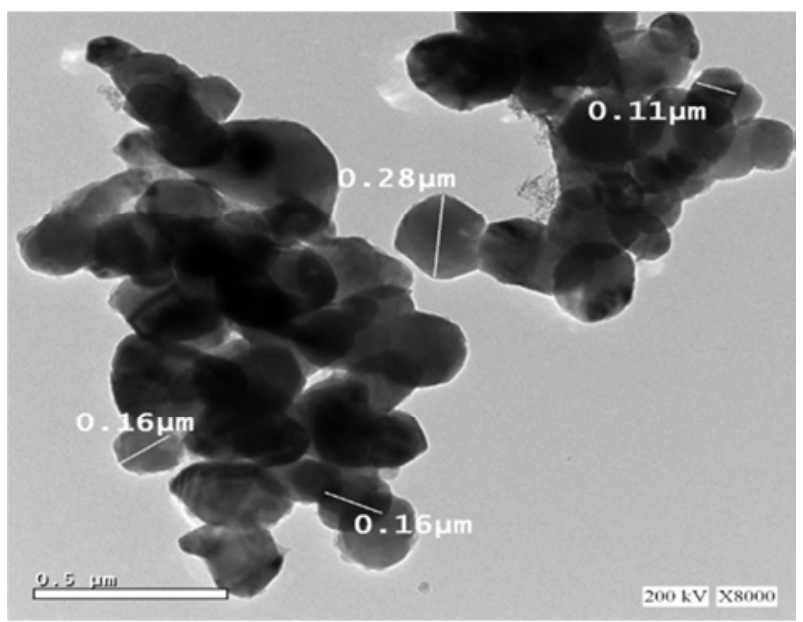

Figure 1. TEM micrographs of $\mathrm{TiO}_{2}$ nanoparticles.

\subsection{FE-SEM Micrographs of XLPE Nanocomposites}

The surface morphology of the samples was analyzed using FE-SEM which can describe the microstructure dispersion of nanoparticles inside the polymer chains. FESEM micrographs for both cross-section area and the surface of all samples were carried out. Figure 2 shows the FE-SEM micrographs for both cross-section area and the surface of the XLPE without nanoparticles.
It is clear that at the same addition ratio of $\mathrm{Tio}_{2}$ there are some aggregates of $\mathrm{TiO}_{2}$ with few micrometers in size within the nonfunctionalized $\mathrm{TiO}_{2}$ but in case of functionalized $\mathrm{TiO}_{2}$ there is a good dispersion and homogenous distribution for the nanoparticles in the composite, that is due to the chemical functionalization process of the $\mathrm{TiO}_{2}$ nanoparticles surface lead 
to strong interactions between nanoparticles and XLPE matrix.
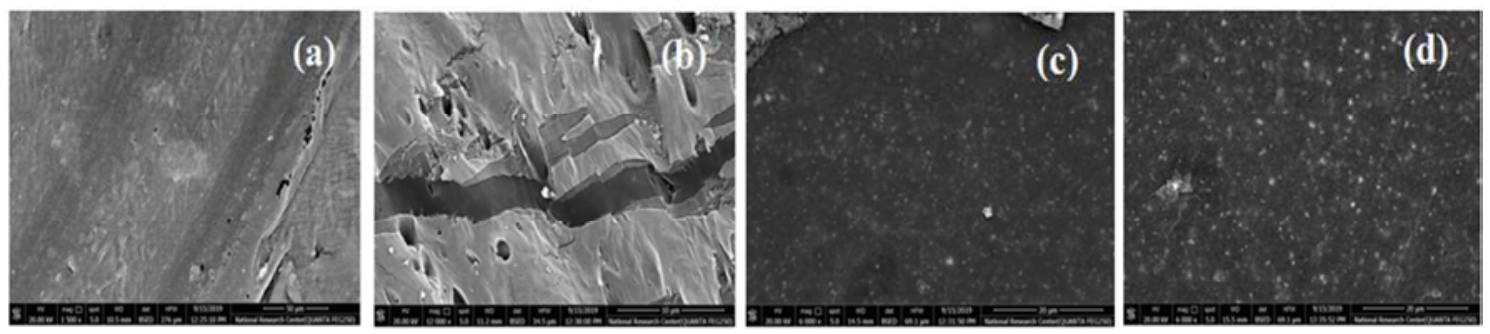

Figure 3. FE-SEM micrographs as cross section area and surface of $X L P E / T i O_{2}$ nanocomposites (a-c) with non-functionalized (b-d) and functionalized TiO ${ }_{2}$.

\subsection{X-ray Diffraction (XRD)}

The X-ray diffraction pattern of the tested XLPE samples indicated an amorphous hallo and two major crystalline peaks at $2 \theta=21.40^{\circ}(\mathrm{d}$ (interplanar distance $)=4.15112 \AA$ ) and $2 \theta=24.06^{\circ}(\mathrm{d}=3.777 \AA)$, Figure $4 \mathrm{a},[30]$. Other small peaks can be observed at $2 \theta=29.8784^{\circ}(\mathrm{d}=2.99052 \AA)$, $2 \theta=39.573^{\circ}(\mathrm{d}=2.27740 \AA), 2 \theta=41.4867^{\circ}(\mathrm{d}=2.17667 \AA)$, $2 \theta=42.8169^{\circ}(\mathrm{d}=2.11207 \AA), 2 \theta=43.7564^{\circ}(\mathrm{d}=2.06888 \AA)$, $2 \theta=46.7704^{\circ}(d=1.94234 \AA), 2 \theta=52.8265^{\circ}(d=1.73306 \AA)$, $2 \theta=54.5422^{\circ}(\mathrm{d}=1.68253 \AA), 2 \theta=57.1883^{\circ}(\mathrm{d}=1.61081 \AA)$, and $2 \theta=61.4206^{\circ}(\mathrm{d}=1.50833 \AA)$.
The major crystalline peaks at $2 \theta=21.40$ and $24.06^{\circ}$ did not change by the addition of $\mathrm{TiO}_{2}$ to XLPE with small changes in the interplanar spacing which recorded a value not more than $(0.006-0.01 \AA)$ with an additional peaks at $2 \theta=27.1779^{\circ}(\mathrm{d}=3.28122 \AA)$ and $2 \theta=68.9025^{\circ}(\mathrm{d}=1.36166 \AA)$ in case of nonfunctionalized and $2 \theta=27.3837^{\circ}(d=3.25702 \AA)$ and $2 \theta=68.9254^{\circ} \quad(d=1.36127 \AA)$ for functionalized $\mathrm{XLPE} / \mathrm{TiO}_{2}$ nanocomposites respectively which represents of crystallization of $\mathrm{TiO}_{2}$ nanoparticles inside the polymer matrix (Figure 4b-c).
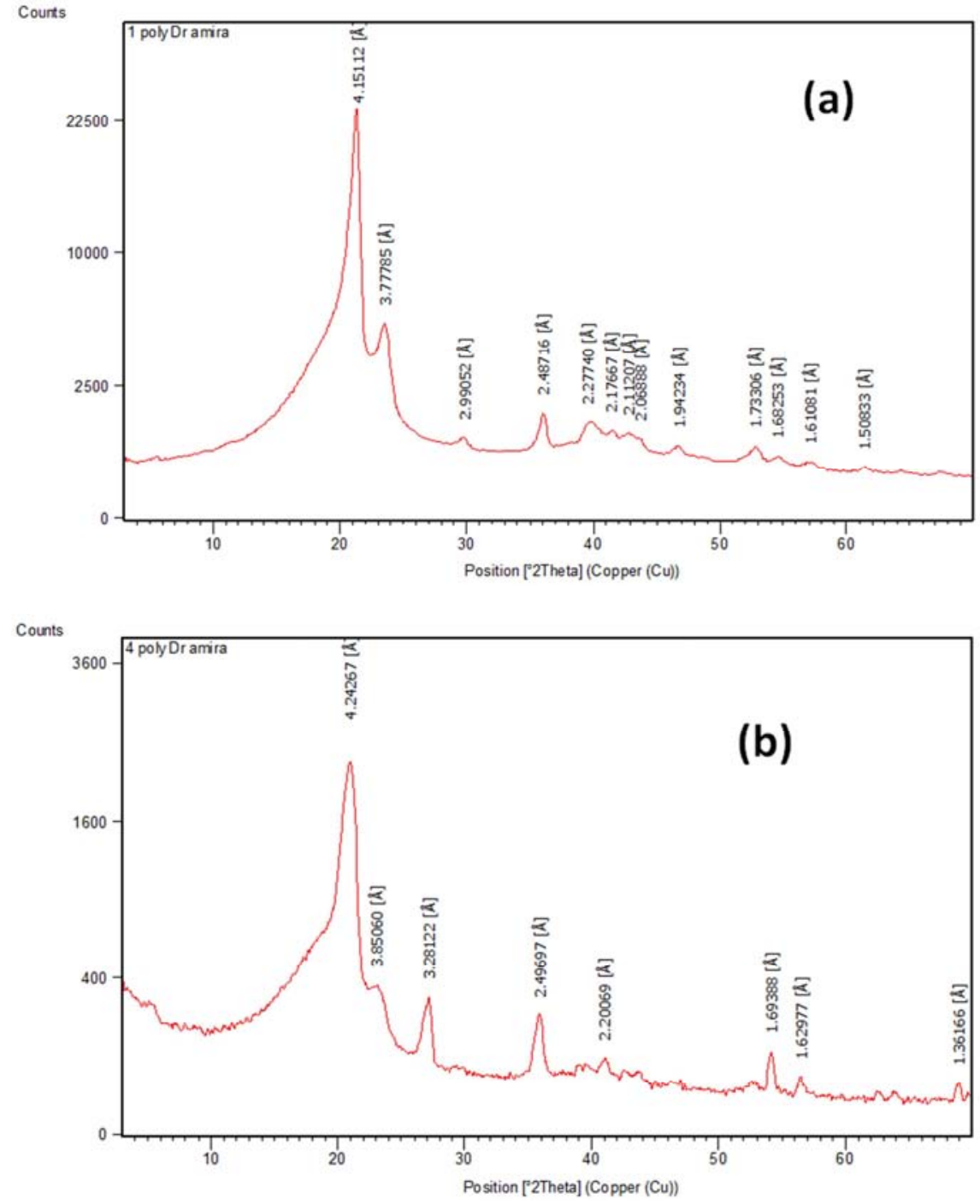


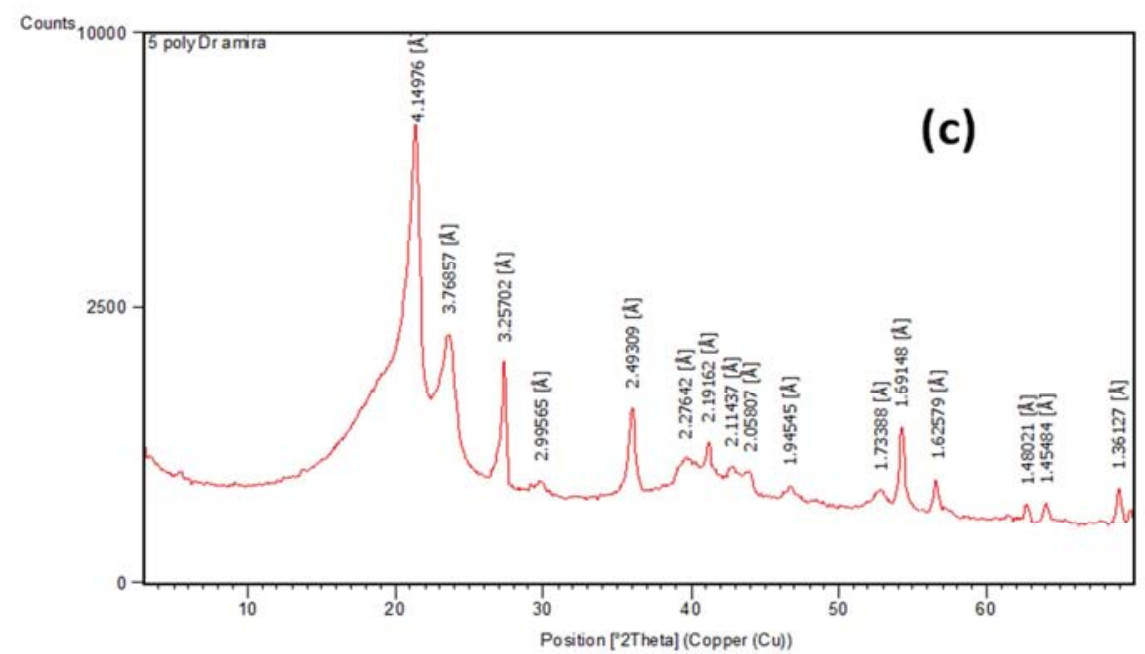

Figure 4. X-ray diffraction patterns of: (a) XLPE, (b) nanocomposites with nonfunctionalized, and (c) functionalized TiO ${ }_{2}$.

\subsection{Thermo-gravimetric Analysis (TGA)}

In TGA analysis, the rate of material weight is changing with varying temperature. The onset of time or temperature of the initial weight loss can be considered as a loss of volatiles, absorbed water or lower molecular weight constituents. The onset of irreversible weight loss could suggest the real thermal degradations [31]. Accordingly, the relative thermal stability of the nanocomposites has been evaluated by comparing their decomposition temperatures. In this study, the weight loss at $300^{\circ} \mathrm{C}, 400^{\circ} \mathrm{C}$, and $475^{\circ} \mathrm{C}$ (decomposition temperature) are the main criteria used to indicate the thermal stability of nanocomposites. The higher the values of decomposition temperatures the higher the thermal stability of the nanocomposites will be [32].

From Figure 5 and Table 1, it is seen that the weight loss of XLPE was $0.83,1.99$, and $62.9 \%$ at 300,400 , and $475^{\circ} \mathrm{C}$. It means that, its rate of loss decreased with increasing temperature. By the addition of nonfunctionalized and functionalized $\mathrm{TiO}_{2}$ nanoparticles the weight loss increased at 300 and $400^{\circ} \mathrm{C}$ while decreased at $475^{\circ} \mathrm{C}$ in case of functionalized $\mathrm{TiO}_{2}$. The decomposition temperature decreased with nonfunctionalized $\mathrm{TiO}_{2}$ and increased with functionalized $\mathrm{TiO}_{2}$. This means the addition of functionalized nanoparticles into the XLPE matrix enhanced the thermal stability of XLPE. This may be due to that the higher thermal resistance of the inorganic filler which has an onset temperature of $580^{\circ} \mathrm{C}$ [33-36] or may be due to the lower mobility of the matrix chains as a result of the strong interaction between the nanoparticles and the XLPE matrix.

Table 1. Weight loss and decomposition temperatures of XLPE and XLPE/TiO $O_{2}$ nanocomposite.

\begin{tabular}{|c|c|c|c|c|}
\hline \multirow{2}{*}{ Sample } & \multicolumn{3}{|c|}{ Weight Loss (\%) } & \multirow{2}{*}{ Decomposition Temperature ${ }^{\circ} \mathrm{C}$} \\
\hline & $300^{\circ} \mathrm{C}$ & $400^{\circ} \mathrm{C}$ & $475^{\circ} \mathrm{C}$ & \\
\hline XLPE & 0.83 & 1.99 & 62.9 & 475.28 \\
\hline XLPE/ Nonfunctionalized $\mathrm{TiO}_{2}$ & 1.02 & 2.34 & 64.07 & 474.7 \\
\hline XLPE/ Functionalized $\mathrm{TiO}_{2}$ & 1.23 & 2.3 & 46.35 & 482.94 \\
\hline
\end{tabular}

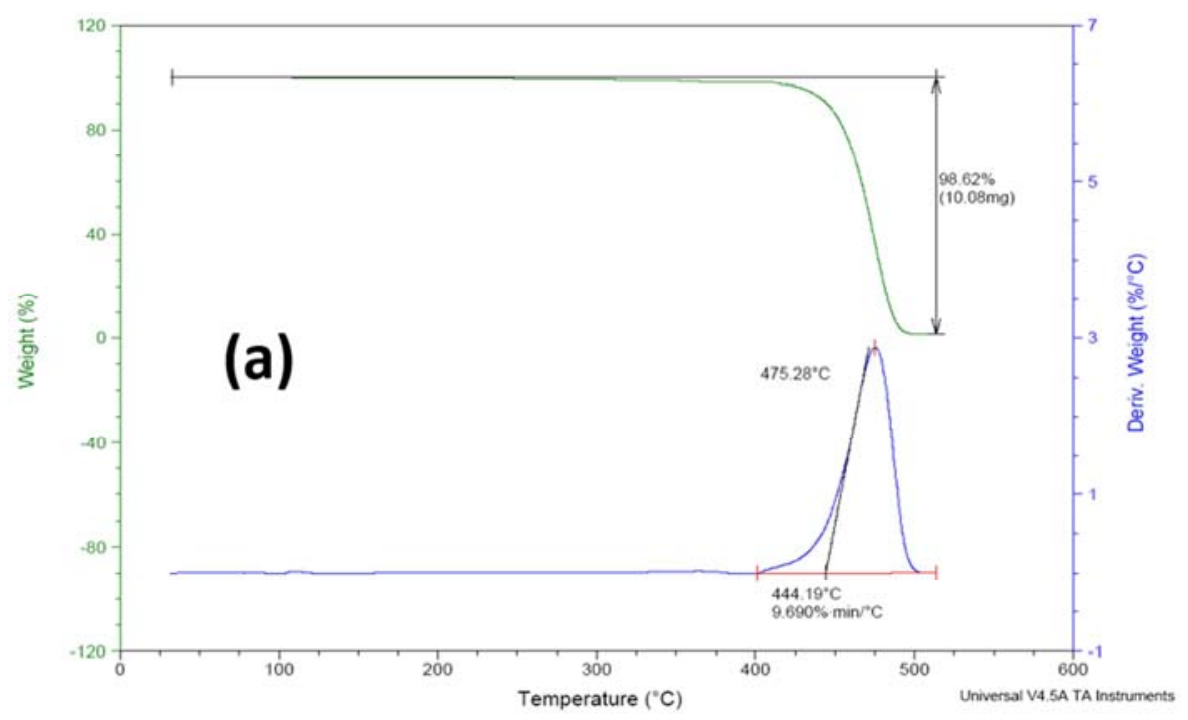



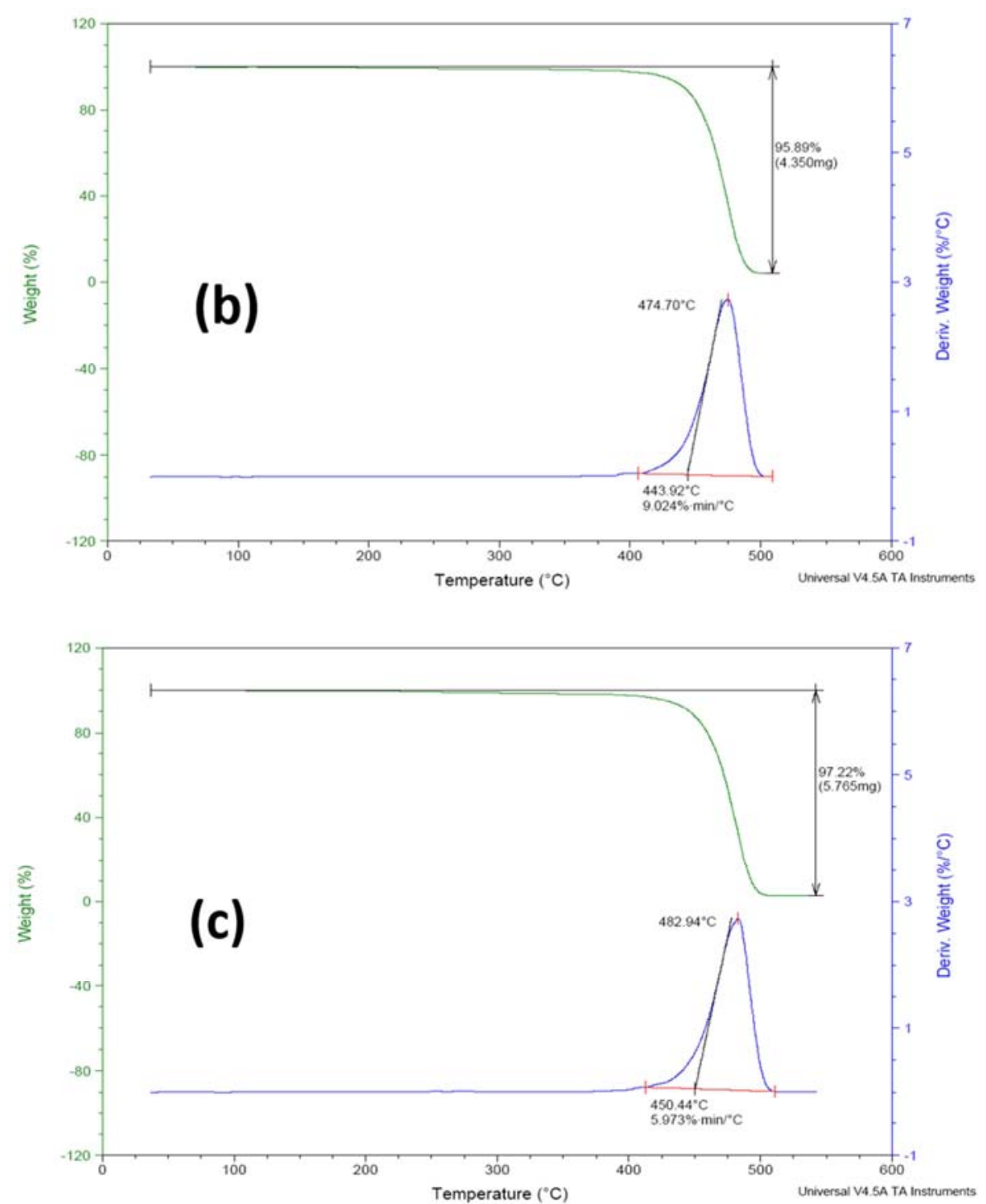

Figure 5. TGA of: (a) XLPE (b) XLPE nanocomposite with nonfunctionalized $\mathrm{TiO}_{2}$ and (c) XLPE nanocomposite with functionalized TiO ${ }_{2}$.

\subsection{Dielectric Properties of XLPE Nanocomposites}

The important dielectric properties, such as the relative permittivity, dielectric loss $(\tan \delta)$ and the dielectric strength were measured. These three parameters measure the quality of the dielectric material used as insulation in HV applications.

\subsubsection{Relative Permittivity and Dielectric Loss}

The relative permittivity and the dielectric loss of the nonfunctionalized and amino-functionalized $\mathrm{XLPE} / \mathrm{TiO}_{2}$ nanocomposites samples were investigated. Figure 6 and Figure 7 show the variation of relative permittivity $\left(\varepsilon_{\mathrm{r}}\right)$ and dielectric loss $(\tan \delta)$ of XLPE and XLPE/TiO nonfunctionalized and functionalized nanocomposites admixed with various amounts of nanoparticles.

It can be concluded that in most samples, with increasing the frequency the diploes do not have enough time to be created in the direction of the electric field. So, the samples did not present high polarizability in high frequencies regions, which leads to a reduction in the relative permittivity values.
However, and in contrast, when the frequency decreases, the time scales will increase and most mobile atom groups begin to orient, resulting in an increase in the observed values of $\varepsilon_{\mathrm{r}}$. Also, it is observed that all prepared samples have relatively high $\tan \delta$ values at low frequencies, and this is may be attributed to the higher leakage current in the system.

Figure $6 \mathrm{a}$ and $7 \mathrm{a}$ show that the values of permittivity of the nanocomposites are varied with the concentration of $\mathrm{TiO}_{2}$ and $\mathrm{XLPE} / \mathrm{TiO}_{2}$ sample with $2 \mathrm{wt} \% \%$ have the lowest $\varepsilon_{\mathrm{r}}$ values especially with the functionalized sample which decreases by about $3.1 \%$ at power frequency $50 \mathrm{~Hz}$. This may be due to the restriction in the polymer chain mobility due to good incorporation and dispersion of the functionalized $\mathrm{TiO}_{2}$ nanoparticles within these chains. This indication of the chemical functionalization of $\mathrm{TiO}_{2}$ assists their dispersion and enhances their compatibility with the polymeric matrix.

Also, it is obvious that both $\mathrm{XLPE} / \mathrm{TiO}_{2}$ nonfunctionalized and amino-functionalized nanocomposites with $5 \mathrm{wt} . \%$ have higher $\varepsilon_{\mathrm{r}}$ values than both neat XLPE since they increased by $15.46 \%$ and $2.061 \%$ respectively at power frequency $50 \mathrm{~Hz}$. 
This increase may be due to the reduction in the interface interaction area between the polymer matrix and nanoparticles that results from nanoparticle agglomeration. These agglomerated nanoparticles may act as voids between the polymer chains and facilitate their mobility, and hence account for more molecular polarization.

Figure $6 \mathrm{~b}$ and $7 \mathrm{~b}$ show the curves of dielectric loss, $\tan \delta$, versus frequencies for different concentration of $\mathrm{TiO}_{2}$ nanocomposites. The figures indicated that $\mathrm{XLPE} / \mathrm{TiO}_{2}$ nanocomposites have high dielectric losses at low frequencies, which may be attributed to the high leakage current in the test system. At the same time, it can be noticed that $\tan \delta$ of all XLPE/TiO ${ }_{2}$ samples recorded lower values than XLPE specimen. Also, the amino-functionalized $\mathrm{XLPE} / \mathrm{TiO}_{2}$ samples have lower values of $\tan \delta$ than nonfunctionalized ones at frequencies up to $1000 \mathrm{~Hz}$. Clearly, at high frequencies, the $\tan \delta$ values were not affected by increasing the amount of $\mathrm{TiO}_{2}$ nanoparticles. The obtained results indicated that at power frequency $(50 \mathrm{~Hz})$, the

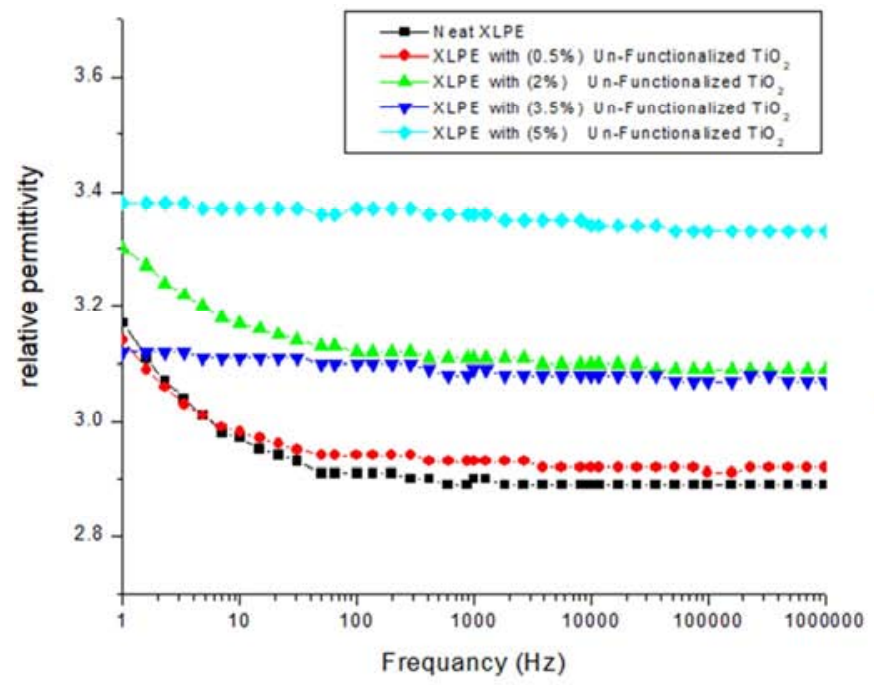

(a) dielectric losses for 2 wt.\% nonfunctionalized $\mathrm{XLPE} / \mathrm{TiO}_{2}$ sample is reduced by $27.34 \%$ over the neat XLPE, which considers the lowest improvement case, and $\tan \delta$ at $5 \mathrm{wt} \%$ amino-functionalized $\mathrm{XLPE} / \mathrm{TiO}_{2}$ sample is reduced by $91.59 \%$, which shows a great improvement, Table 2 .

Table 2. Permittivity and dielectric loss of XLPE and XLPE/TiO nanocomposites at $50 \mathrm{~Hz}$.

\begin{tabular}{|c|c|c|c|}
\hline \multirow{2}{*}{ Sample } & \multirow{2}{*}{ Wt.\% } & Relative permittivity & Loss Factor \\
\hline & & $\left(\varepsilon_{\mathrm{r}}\right)$ & $(\tan \delta)$ \\
\hline \multirow[t]{2}{*}{ XLPE } & 0 & 2.91 & 0.0113 \\
\hline & 0.5 & 2.94 & 0.0081 \\
\hline XLPE / & 2 & 3.13 & 0.00821 \\
\hline Nonfunctionalized & 3.5 & 3.1 & 0.00292 \\
\hline \multirow[t]{2}{*}{$\mathrm{TiO}_{2}$} & 5 & 3.36 & 0.00189 \\
\hline & 0.5 & 2.91 & 0.00276 \\
\hline \multirow{3}{*}{$\begin{array}{l}\text { XLPE/ } \\
\text { Functionalized } \mathrm{TiO}_{2}\end{array}$} & 2 & 2.82 & 0.00190 \\
\hline & 3.5 & 2.94 & 0.00138 \\
\hline & 5 & 2.97 & 0.00095 \\
\hline
\end{tabular}

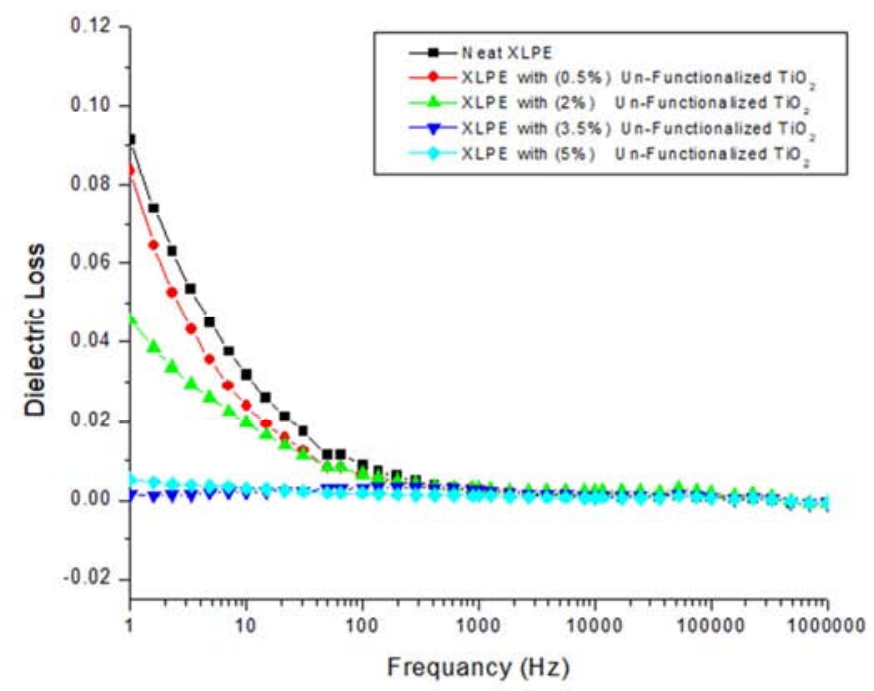

(b)

Figure 6. XLPE and nonfunctionalized XLPE/TiO 2 nanocomposites (a) Relative permittivity, (b) dielectric loss.

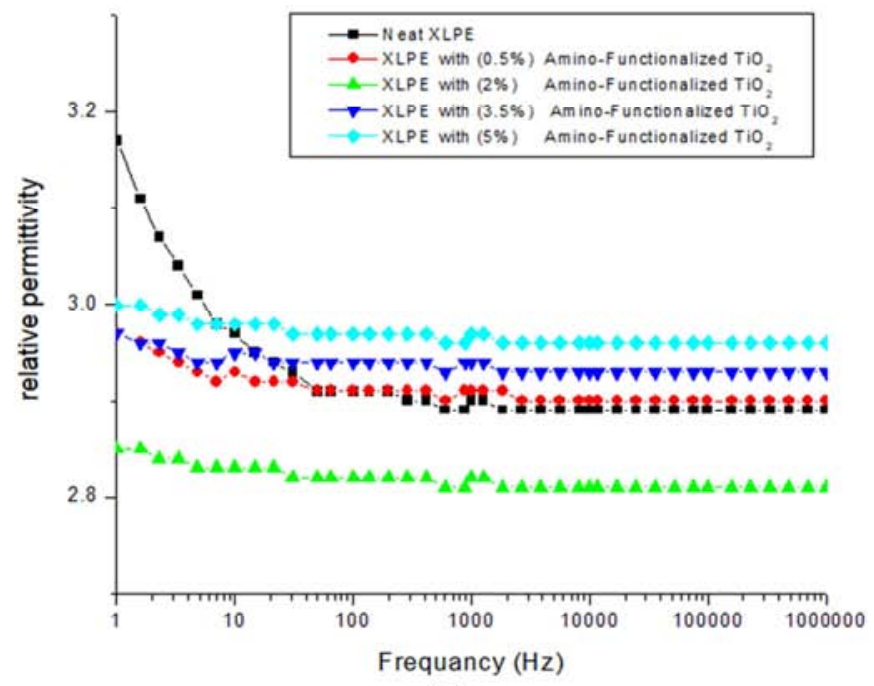

(a)

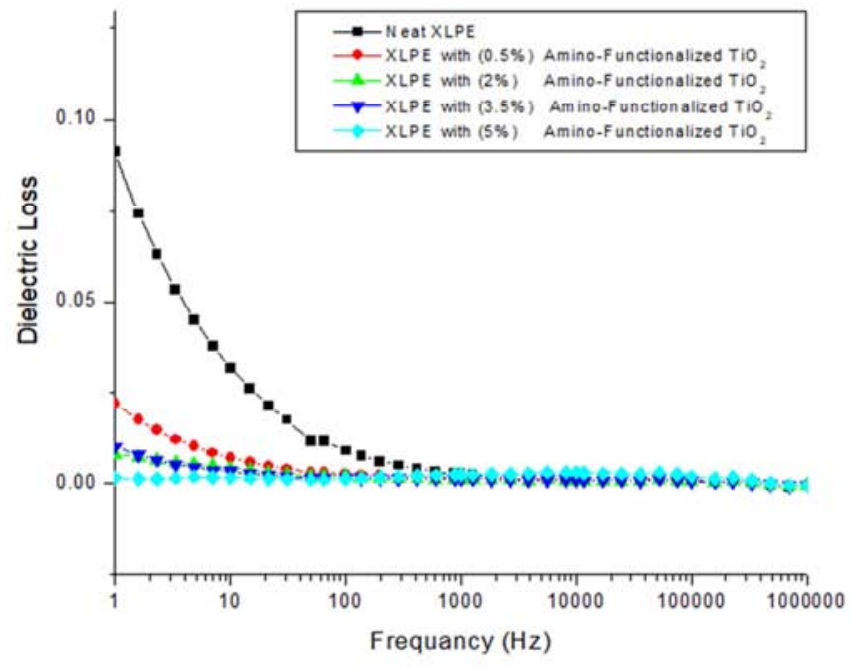

(b)

Figure 7. XLPE and amino-functionalized $X L P E / T i O_{2}$ nanocomposites (a) Relative permittivity, (b) dielectric loss. 


\subsubsection{AC Breakdown Strength}

The AC breakdown voltages (AC-BDV) of XLPE/TiO nanocomposites were measured. Table 3 shows the measured values of $\mathrm{AC}$ breakdown strength of functionalized $\mathrm{XLPE} / \mathrm{TiO}_{2}$ and the nonfunctionalized $\mathrm{XLPE} / \mathrm{TiO}_{2}$ and the percentage of enhancement. The table shows that the functionalized $\mathrm{XLPE} / \mathrm{TiO}_{2}$ nanocomposites are higher than that of the nonfunctionalized ones. This can be attributed to the creation of a strong interfacial area between nanoparticles and the polymeric matrix. In addition, by increasing the addition ratio of nanofiller, the AC-BDS is increased, except at weight fractions of 3.5 wt. $\%$ and 5 wt. $\%$ of $\mathrm{TiO}_{2}$ nanoparticles, the AC-BDS began to reduce. The addition of $\mathrm{TiO}_{2}$ into the XLPE matrix can increase the AC-BDS, while with a high concentration of nanoparticles a lot of agglomeration maybe produced and cause a reduction in ACBDS. Moreover, the maximum measured values of AC-BDS are happen in the samples of XLPE/TiO ${ }_{2}$ with $2 \mathrm{wt} . \%$ and 3.5 wt. $\%$ amino-functionalized $\mathrm{TiO}_{2}$ nanocomposites as shown in Figure 8 , which are enhanced by $24.5 \%$ and $20.08 \%$, respectively, over XLPE. Otherwise, for nonfunctionalized samples, there is no enhancement was observed.

The AC-BDS was obtained using a simulation model on Femm package, which uses the finite element method in 2dimensional pattern (i.e. an axisymmetric form). The voltage applied to the upper sphere is the breakdown voltage taken from the experimental result, and the lower sphere is grounded. The voltage and electric field distribution inside and around the samples are traced and evaluated. The breakdown strength at the tip point of the $\mathrm{HV}$ sphere was recorded as in a Table 3 , in case of XLPE/nonfunctionalized $\mathrm{TiO}_{2}$ and XLPE/aminofunctionalized $\mathrm{TiO}_{2}$ nanocomposites. The electric potential and electrostatic field distribution in and around the sample are shown in Figure 9a and Figure $9 \mathrm{~b}$ for XLPE nanocomposite with 2 wt.\% amino-functionalized nanoparticles. It is shown that there is a high agreement between the measured and the simulated results, where the maximum enhancement of simulation results happened at 2 wt.\% amino-functionalized XLPE/TiO ${ }_{2}$ sample.

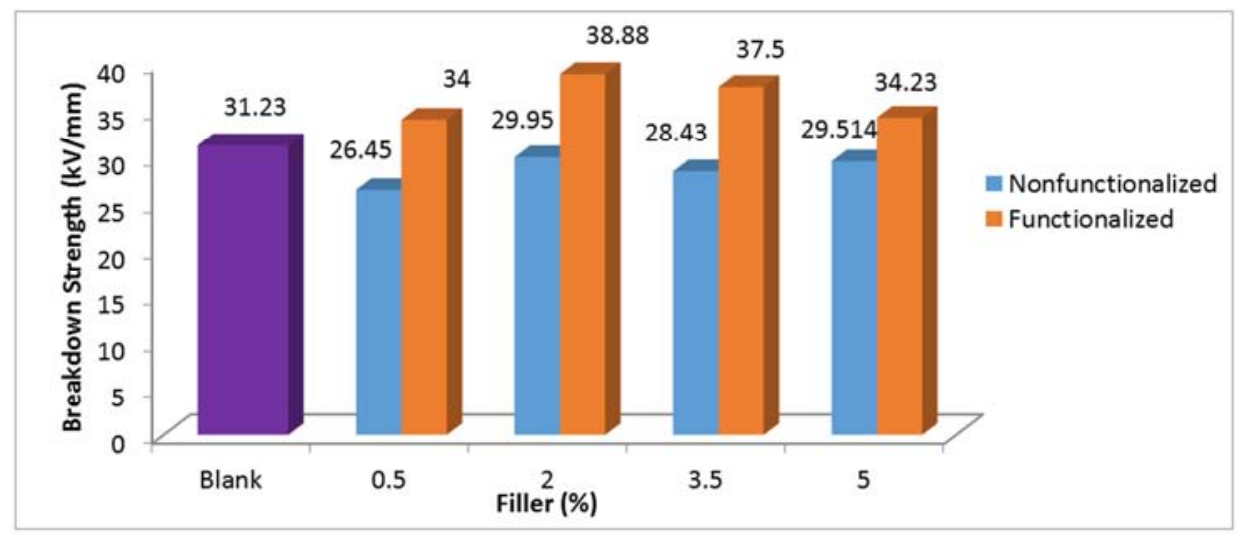

Figure 8. AC-BDS rms values of $X L P E / T_{i} O_{2}$ nanocomposites.

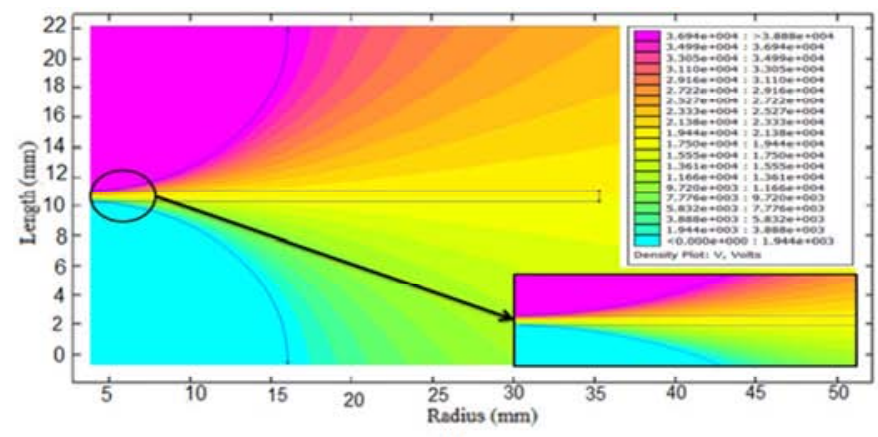

(a)

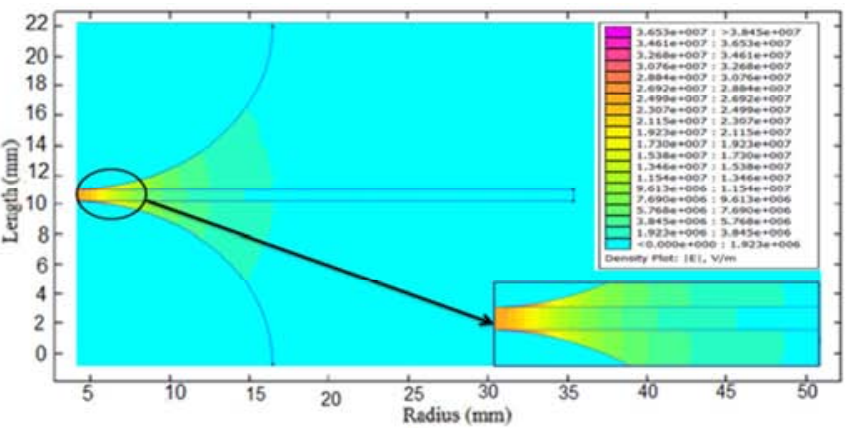

(b)

Figure 9. (a) AC potential distribution around sphere electrode and along with sample thickness, (b) Electrostatic field distribution inside 2 wt.\% XLPE/aminofunctionalized $\mathrm{TiO}_{2}$ nanocomposite for the sphere to sphere configuration.

Table 3. Measured and simulated rms values of $A C-B D S$ of the $X D P E / T i O_{2}$ nanocomposites at sphere-sphere field.

\begin{tabular}{|c|c|c|c|c|}
\hline \multirow{2}{*}{ Sample } & Wt. & Breakdown strength & Improvement & Simulated Breakdown strength \\
\hline & $(\%)$ & $(\mathrm{kV} / \mathrm{mm})$ & $(\%)$ & $(\mathrm{kV} / \mathrm{mm})$ \\
\hline \multirow[t]{2}{*}{ XLPE } & 0 & 31.23 & 0 & 32.64 \\
\hline & 0.5 & 26.45 & $-15.30 \%$ & 27.6 \\
\hline \multirow{3}{*}{ XLPE/ Nonfunctionalized $\mathrm{TiO}_{2}$} & 2 & 29.95 & $-4.10 \%$ & 31.46 \\
\hline & 3.5 & 28.43 & $-8.97 \%$ & 30.03 \\
\hline & 5 & 29.514 & $-5.49 \%$ & 30.096 \\
\hline
\end{tabular}




\begin{tabular}{lllll}
\hline \multirow{2}{*}{ Sample } & Wt. & Breakdown strength & Improvement & Simulated Breakdown strength \\
\cline { 2 - 5 } & $\mathbf{( \% )}$ & $\mathbf{( k V / m m )}$ & $\mathbf{( \% )}$ & $\mathbf{( k V / m m )}$ \\
\hline & 0.5 & 34 & $8.87 \%$ & 35.108 \\
XLPE/ Functionalized TiO2 & 2 & 38.88 & $24.50 \%$ & 40.401 \\
& 3.5 & 37.5 & $20.08 \%$ & 38.76 \\
& 5 & 34.23 & $9.61 \%$ & 35.49 \\
\hline
\end{tabular}

\subsection{Mechanical Properties}

Tensile strength and elongation level measurement was carried for XLPE with nonfunctionalized and functionalized $\mathrm{TiO}_{2}$ at different wt.\%. Figure 10 shows tensile strength for XLPE was $10 \mathrm{MPa}$ and 584 elongations. For nonfunctionalized $\mathrm{XLPE} / \mathrm{TiO}_{2}$ nanocomposites, it can be ascertained from the Figure 10 that the tensile strength is increased with increasing the wt. $\%$ and the maximum value was at $3.5 \%$ wt. which reaches $11.6 \mathrm{MPa}$ and the elongation was 450 , then with increasing the wt.\%, tensile strength decreases reaching 10.5 $\mathrm{MPa}$ at 5 wt.\% and 394 in elongation. This behavior can be explained as follow, in case of high loading of nonfunctionalized $\mathrm{TiO}_{2}$, the agglomeration of the nanoparticles acts as relatively large voids in the tested sample which make the molecular chains moving freely and slipping past one another

For functionalized $\mathrm{XLPE} / \mathrm{TiO}_{2}$ nanocomposites, it can be ascertained that the tensile strength is maximum at $3.5 \mathrm{wt} . \%$ which reaches $12.4 \mathrm{MPa}$ and the elongation value was $460 \%$ then with increasing the wt.\%, tensile strength decreases reaching $11 \mathrm{MPa}$ at $5 \mathrm{wt} \%$ with elongation $402 \%$. When compare to blank sample, 3.5 wt. $\%$ of functionalized titanium dioxide has more tensile strength and more elongation. These results emphasis that $\mathrm{TiO}_{2}$ functionalized nanoparticles improved the mechanical characteristics of XLPE sample. Such behavior in mechanical properties and the increase in the tensile strength with low loading of nanoparticle are resulted from their high specific surface available for interactions with the polymer matrix. For many polymers, addition of nanofiller causes a notable diminution of chain elongation, this could be attributed to the constraint of the chains mobility generated by creating a bonding between nanoparticles and polymer chains [37].
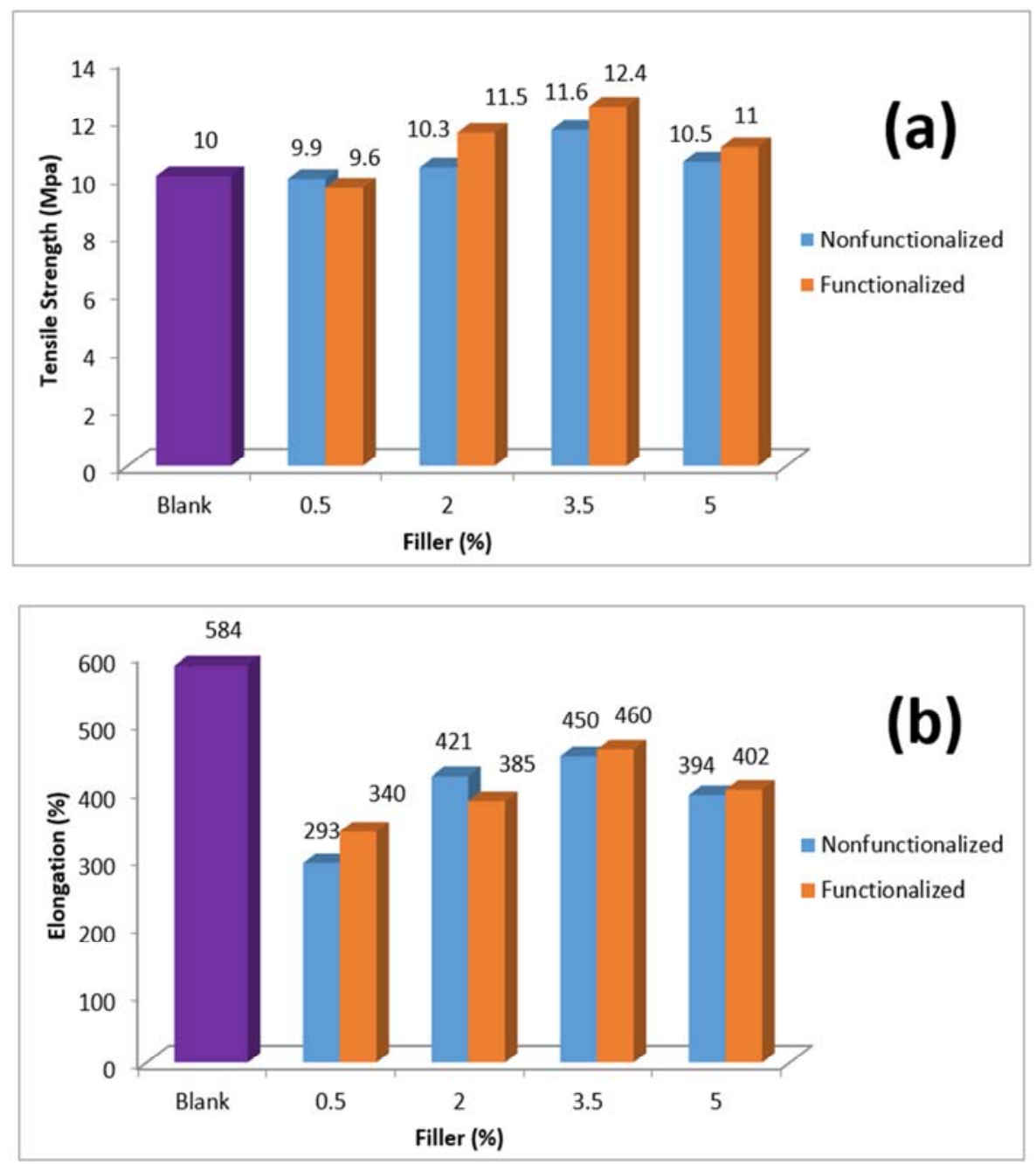

Figure 10. Tensile strength (a), and elongation (b) of nonfunctionalized and functionalized $\mathrm{TiO}_{2} / X L P E$ nanocomposites with different weight ratios. 


\section{Conclusion}

The addition of inorganic nanoparticles into the XLPE matrix enhanced the thermal and mechanical properties of XLPE. The functionalization nanoparticles lead to an excellent dispersion within polymer chains. An improvement in relative permittivity and loss tangent, where the loss tangents for all XLPE/TiO 2 nanocomposites were less than that of the XLPE. The dielectric loss has a higher value in the low-frequency regions, where the interface polarization in XLPE nanocomposites is significant. The breakdown strength of the modified nanocomposites has higher values compared to blank (neat XLPE). As the percentage weight of nanoparticles increased, the interparticle distance decreases, which leads to a hindrance in charge carriers transferring and create a strong bond, which resulted in increasing the dielectric strength. However, it is noticed that more addition of $\mathrm{TiO}_{2}$ nanoparticles decreases the dielectric strength and that's maybe due to the high concentration of nanoparticles leads to more agglomeration causing much reduction in $\mathrm{AC}$ BDS.

\section{References}

[1] King, A., Wentworth, V. H.: Raw materials for electric cables. Benn (1954).

[2] Maddah Hisham, A.: Polypropylene as a promising plastic. A review. Am. J. Polym. Sci. 6, 1-11 (2016).

[3] Hyvonen, P.: Prediction of insulation degradation of distribution power cables based on chemical analysis and electrical measurements. Teknillinen korkeakoulu (2008).

[4] Hanley, T. L., Burford, R. P., Fleming, R. J., Barber, K. W.: A general review of polymeric insulation for use in HVDC cables. IEEE Electrical Insulation Magazine. 19, 13-24 (2003).

[5] Tamboli, S. M., Mhaske, S. T., Kale, D. D.: Crosslinked polyethylene (2004).

[6] Rajput, N.: Methods of preparation of nanoparticles-a review. Inter. J. Adv. Engin and Tech. 7, 1806 (2015).

[7] Jeon, I. Y.; Baek, J. B. Nanocomposites derived from polymers and inorganic nanoparticles. Materials. 3, 3654-3674. (2010)/

[8] Abdel-Gawad, N. M., El Dein, A. Z., Mansour, D. E. A., Ahmed, H. M., Darwish, M. M. F., Lehtonen, M.: Multiple enhancement of PVC cable insulation using functionalized $\mathrm{SiO}_{2}$ nanoparticles based nanocomposites. Electric Power Systems Research. 163, 612-625 (2018)

[9] Abdel-Gawad, N. M., El Dein, A. Z., Mansour, D. E. A., Ahmed, H. M., Darwish, M. M. F., Lehtonen, M.: Enhancement of dielectric and mechanical properties of polyvinyl chloride nanocomposites using functionalized $\mathrm{TiO}_{2}$ nanoparticles. IEEE Transactions on Dielectrics and Electrical Insulation. 24, 3490-3499 (2017).

[10] Abdel-Gawad, N. M., El Dein, A. Z., Mansour, D. E. A.; Ahmed, H. M., Darwish, M. M. F., Lehtonen, M.: Development of industrial scale PVC nanocomposites with comprehensive enhancement in dielectric properties. IET Sci, Measurement and Tech. 13, 90-96 (2018).

[11] Qingyue, Y., Xiufeng, L., Peng, Z., Peijie, Y., Youfu, C.: Properties of Water Tree Growing in XLPE and composites, International Conference on Electrical Materials and Power Equipment. (ICEMPE). IEEE. 409-412 (2019)

[12] Salh S. H., Raswl D. A.: Thermal stability of polymer composite films based on polyvinyl alcohol doped with different fillers. Open Access Journal of Physics. 2, 5-10 (2018).

[13] Awad A. H., El-Wahab, A. A. A., El-Gamsy, R., Abdel-latif, M. H.: A study of some thermal and mechanical properties of HDPE blend with marble and granite dust. Ain Shams Engineering Journal. 10, 353-358 (2019).

[14] Giżyński, M., Romelczyk-Baishya, B.: Investigation of carbon fiber-reinforced thermoplastic polymers using thermogravimetric analysis. Journal of Thermoplastic Composite Materials. 1-15 (2019).

[15] Zhang, C., Chang, J., Zhang, H., Li, C., Zhao, H. Improved Direct Current Electrical Properties of Crosslinked Polyethylene Modified with the Polar Group Compound. Polymers. 11, 1624 (2019).

[16] Liu, S. H., Shen, M. Y., Kuan, C. F., Kuan, H. C., Ke, C. Y., Chiang, C. L.: Improving Thermal Stability of Polyurethane through the Addition of Hyperbranched Polysiloxane. Polymers. 11, 697 (2019).

[17] Liu, Z., Tu, R., Liao, Q., Hu, H., Yang, J., He, Y., Liu. W.: High thermal conductivity of flake graphite reinforced polyethylene composites fabricated by the powder mixing method and the melt-extruding process. Polymers. 10, 693 (2018).

[18] Chi, X., Cheng, L., Liu, W., Zhang, X., Li, S.: Characterization of polypropylene modified by blending elastomer and nano-silica. Materials. 11, 1321 (2018).

[19] Helal, E., Pottier, C., David, E., Fréchette, M., Demarquette, N. R. E.: Polyethylene/thermoplastic elastomer/Zinc Oxide nanocomposites for high voltage insulation applications: Dielectric, mechanical and rheological behavior. European Polymer Journal. 100, 258-269 (2018).

[20] Gong, J., Hosaka, E., Sakai, K., Ito, H., Shibata, Y., Sato, K., Hamada, K.: Processing and thermal response of temperaturesensitive-gel (TSG)/polymer composites. Polymers. 10, 486 (2018).

[21] Khan, I., Saeed, K., Khan, I.: Nanoparticles: Properties, applications and toxicities. Arabian Journal of Chemistry. 12, 908-931 (2019).

[22] Khodaparast, P, Ounaies Zoubeida.: On the impact of functionalization and thermal treatment on dielectric behavior of low content $\mathrm{TiO}_{2}$ PVDF nanocomposites. IEEE Transactions on Dielectrics and Electrical Insulation. 20, 166167 (2013).

[23] Kubacka, A., Fernández-García, M., Cerrada, M. L., Fernández-García, M.: Titanium Dioxide-Polymer Nanocomposites with Advanced Properties. In: NanoAntimicrobials. Springer, Berlin, Heidelberg. 119-149 (2012). 
[24] Dalod, A. R., Henriksen, L., Grande, T., Einarsrud, M. A.: Functionalized $\mathrm{TiO}_{2}$ nanoparticles by single-step hydrothermal synthesis, the role of the silane coupling agents. Beilstein journal of nanotechnology. 8, 304-312 (2017).

[25] Chuang, W., Geng-sheng, J., Lei, P.; Bao-lin, Z., Ke-zhi, L., Jun-long, W.: Influences of surface modification of nano-silica by silane coupling agents on the thermal and frictional properties of cyanate ester resin. Results in Physics. 9, 886896 (2018).

[26] Zhao, J., Milanova, M., Warmoeskerken, M. M., Dutschk, V.: Surface modification of $\mathrm{TiO}_{2}$ nanoparticles with silane coupling agents. Colloids and surfaces A. Physicochemical and engineering aspects. 413, 273-279 (2012).

[27] Prado, L. A., Sriyai, M., Ghislandi, M., Barros-Timmons, A., Schulte, K.: Surface modification of alumina nanoparticles with silane coupling agents. Journal of the Brazilian Chemical Society. 21, 2238-2245 (2010).

[28] A bdel-Gawad, N. M., Mansour, D. E. A., El Dein, A. Z., Ahmed, H. M., Darwish, M. M. F.: Effect of functionalized $\mathrm{TiO}_{2}$ nanoparticles on dielectric properties of PVC nanocomposites used in electrical insulating cables. In Eighteenth International Middle East Power Systems Conference (MEPCON). IEEE. 693-698 (2016).

[29] Ahn, S. H., Kim, S. H., Lee, S. G.: Surface-modified silica nanoparticle-reinforced poly (ethylene 2, 6-naphthalate), Journal of Applied Polymer Science. 94, 812-818 (2004).

[30] Hedir, A., Moudoud, M., Lamrous, O., Rondot, S., Jbara, O., Dony, P.: Ultraviolet radiation aging impact on physicochemical properties of crosslinked polyethylene cable insulation, J. applied polymer sci. 137, 48575 (2020).

[31] Juliana, N. C., Chibuike, N. A. O., Josiah, E. A.: Evaluation of the Thermal Stability of Poly (O-phenylenediamine) (PoPD) by Thermogravimetric Analysis (TGA). American J. Nanosci. $5,18-22(2019)$.

[32] El-Sayed, N. S., El-Sakhawy, M.; Hesemann, P., Brun, N., Kamel, S.: Rational design of novel water-soluble ampholytic cellulose derivatives. Inter. J. biol. Macromol. 114, 363-372 (2018).

[33] Huang, C., Qian, X., Yang, R.: Thermal conductivity of polymers and polymer nanocomposites. Materials Science and Engineering, R: Reports. 132, 1-22 (2018).

[34] Khan, H., Amin, M., Ali, M., Iqbal, M., Yasin, M.: Effect of micro/nano- $\mathrm{SiO}_{2}$ on mechanical, thermal, and electrical properties of silicone rubber, epoxy, and EPDM composites for outdoor electrical insulations. Turkish Journal of Electrical Engineering \& Computer Sciences. 25, 1426-1435 (2017).

[35] Corcione, C. E., Frigione, M.: Characterization of nanocomposites by thermal analysis. Materials. 5, 2960-2980. (2012).

[36] Nabinejad, O., Sujan, D., Rahman, M. E., Davies, I. J.: Determination of filler content for natural filler polymer composite by thermogravimetric analysis. J. Thermal Analas. And Calorim. 122, 227-233 (2015).

[37] Q. OYU, A. P. S. SELVADURA: Mechanical behaviour of a plasticized PVC subjected to ethanol exposure. Polymer degradation and stability. 89 (1), 109-124 (2005). 